\title{
Meta-model based optimization of a large diameter semi-radial conical hub engine cooling fan
}

\author{
Z. Zhang ${ }^{1,2, a}$, M. Buisson ${ }^{1}$, P. Ferrand ${ }^{1}$, M. Henner $^{2}$ And F.Gillot ${ }^{3}$ \\ 1 Université de Lyon, UMR CNRS 5509, Laboratoire de Mécanique des Fluides et d'Acoustique, École Centrale de Lyon, \\ 69130 Ecully, France \\ 2 Valeo Systèmes Thermiques, 8 rue Louis Lormand, 78321 La Verriere, France \\ 3 Université de Lyon, UMR CNRS 5513, Laboratoire de Tribologie et Dynamique des Systèmes, École Centrale de Lyon, \\ 69130 Ecully, France
}

Received 18 June 2013, Accepted 25 June 2014

\begin{abstract}
Turbomachinery design is an iterative process that can be time-consuming and expensive, especially when an extensive knowledge of the performance envelope is required. The approach described in the present paper can significantly cut the turnaround times down without jeopardizing the accuracy of the final result. A parameterization technique based on radial basis functions (RBF) is used and Reynolds Averaged Navier-Stokes (RANS) simulations are subsequently performed on a set of selected morphed meshes, the goal of which is to produce an aerodynamic database containing first-order, second-order and second-order cross derivatives of objectives with respect to parameters. New solutions, corresponding to any variations of the selected parameters, can thus be extrapolated thanks to the information included in the aforementioned database. In this way, a meta-model is built and can be easily explored by a genetic algorithm. This approach has been experimented on a new concept of engine cooling fan featuring low torque and high efficiency. A reference fan design has been adapted for the particular surrounding of the vehicle underhood, where the downstream flow is radially deviated from its axis by the engine. The optimization process has resulted in an efficiency improvement of three points for one of the obtained optima.
\end{abstract}

Key words: Turbomachinery design / computational fluid dynamics (CFD) / parameterization / mesh morphing / meta-model / optimization / engine cooling fan

\section{Study context and reference design}

Fan systems are used in automotive engine cooling modules to increase air flow rate through heat exchangers. These latters are dedicated to the thermal management of the vehicle, for both the engine (radiator and charge air cooler) and the cabin climate control.

The willing to decrease power consumption of electrical accessories in the vehicle incites to enhance fan system efficiency and numerous studies have been performed towards this goal. Among them, an investigation [1] aimed at reducing the losses produced by the tip inverse flow, which is found between the shroud and the rotating ring attached to the blade tip. However, modifications in the blade load distribution called for an additional study [2], and performance improvements were finally observed after a blade tip readjustment. Although the rotating ring

${ }^{a}$ Corresponding author: zebin.zhang@ec-lyon.fr is frequently used to limit tip flow recirculation, its contribution to shear losses is significant and might be a disadvantage to achieve low-torque design. Therefore, investigations focused on a new type of fan design without external ring.

A conical hub has also been integrated to the new geometry in order to produce a down-stream semi-radial flow. This particularity is more suitable for vehicle underhood conditions, where the flow is radially deviated by the engine. For a conventional cylindrical hub fan, it has been observed that the lower radius part of the fan blade contributes very little to the guidance of the air flow through the vein in the blade-to-blade passage. In fact, a local separation is often seen near the hub, which partially blocks the main flow and brings significant losses. This is mainly due to the fact that the downstream flow creates a stagnation region, and consequently an adverse pressure gradient. This phenomenon is caused by the car engine which creates a downstream blockage. As a conical hub 


\section{Nomenclature}

\begin{tabular}{|ll|}
\hline$\eta_{s}$ & Static efficiency \\
$\gamma$ & Stagger angle \\
$\gamma_{\text {hub }}$ & Hub section stagger angle \\
$\gamma_{\text {ref }}$ & Reference stagger angle \\
$\gamma_{\text {tip }}$ & Tip section stagger angle \\
$\psi$ & Sweep parameter \\
$\Delta \gamma_{\text {max }}$ & Maximum stagger angle variation \\
$\Delta \gamma_{\text {min }}$ & Minimum stagger angle variation \\
$p$ & Parameter variable \\
$Q$ & Volume flow rate \\
$r_{\text {hub }}$ & Hub section radius \\
$r_{\text {tip }}$ & Tip section radius \\
$P_{s}{ }^{\text {in }}$ & Static pressure at inlet \\
$\Delta \theta$ & Sweep angle variation \\
$\Delta p$ & Parameter variation \\
$\Delta P_{s}$ & Static pressure rise \\
$\Delta r$ & Radius variation \\
$\overrightarrow{e_{r}}$ & Radial unit vector \\
$\vec{F}_{p}$ & Pressure force \\
$\vec{F}_{v}$ & Viscous force \\
$F$ & Objective variable \\
$P_{s}$ out & Static pressure at outlet \\
$N$ & Rotational speed \\
rpm & Rotations per minute \\
$T$ & Torque \\
\hline
\end{tabular}

can effectively relax this situation by deflecting the fluid radially, a first design with such feature was previously proposed as a proof-of-concept. Three innovative points compared with a traditional design were integrated:

- a conical hub which, as explained previously, drives the flow radially and provides the fluid with more energy;

- the absence of tip ring makes the fan wetted area smaller and therefore reduces the contribution of viscous forces in the overall torque. A gain in efficiency is not only expected but lower cost and lower consumption of the electrical motor could also be achieved;

- a reduced number of blades is also in favor of a lower torque and leads to the same benefits as the previous point.

The current study presents the methodology used to perform an optimization of this semi-radial wheel. It should be noted that the reference design comes from a previous optimization but based on a limited number of parameters and on limited ranges. The decision to carry out a new optimization process based on an extended design space has therefore been made. The reference configuration is characterized by:

- an upstream hub radius of $110 \mathrm{~mm}$;

- a downstream hub radius of $132 \mathrm{~mm}$;

- a hub chord length of $104 \mathrm{~mm}$;

- a tip chord length of $81 \mathrm{~mm}$;

- a tip radius of $212 \mathrm{~mm}$;
- a tip gap of $3 \mathrm{~mm}$;

- a flow rate of $2300 \mathrm{~m}^{3} \cdot \mathrm{h}^{-1}$;

- a rotation speed of $2800 \mathrm{rpm}$.

Four consecutive steps are used in the proposed methodology:

- a continuous unstructured mesh deformation tool Turb'Mesh is used to morph the mesh through userdefined geometric parameters;

- numerical simulations are performed using an incompressible pressure based RANS finite volume CFD solver SC/Tetra, for which Menter's Shear Stress Transport (SST) turbulence model is used;

- a meta-model is built thanks to high-order Taylor series expansion making the most of an aerodynamic derivative database;

- Non-Dominated Sorting Genetic Algorithm 2 (NSGA-2) is coupled with the meta-model and explores the design space.

\section{Geometric parameters and mesh deformation}

Four parameters are considered to morph the reference design and its associated mesh.

Firstly, three classical parameters stagger angles at hub $\left(\gamma_{\text {hub }}\right)$, mid-span $\left(\gamma_{\text {mid }}\right)$ and tip $\left(\gamma_{\text {tip }}\right)$ are chosen.

Figure 1 shows on the left the three stagger angles on a perspective view of the fan. The sketch on the right illustrates the stagger angle variation on a blade-to-blade section. The reference stagger angle $\gamma_{\text {ref }}$ is depicted by the angle between the chord line (black solid line) and the axis of rotation (dash-dotted line which corresponds to the $x$-axis on the left figure). Assuming center of rotation to be the leading edge of the $2 \mathrm{D}$ profile, a rotation of the chord line towards the green dashed line results in a positive variation of the stagger angle, which decreases the incidence and unloads the blade, whereas a negative value is taken when the chord line rotates towards the red dashed line, and the blade is consequently higher loaded.

Additionally, a user-defined parameter "sweep $(\psi)$ " is taken into account. This parameter is defined to modify simultaneously three sweep angles at hub, mid-span and tip. This is achieved by the following function.

$$
\Delta \theta=\psi\left(1-6 \Delta r^{2}+4 \Delta r^{3}\right)
$$

The function (Eq. (1)) is a third order polynomial function, where " $\psi$ " is the sweep parameter, which controls all the sweep angles from hub to tip. $\Delta \theta$ is the sweep angle at a radius $r, \Delta r$ is defined by:

$$
\Delta r=\frac{r-r_{\mathrm{hub}}}{r_{\mathrm{tip}}}
$$

where $r_{\text {hub }}$ is the radius at hub section and $r_{\text {tip }}$ is the radius at tip section. $\Delta r=0$ indicates the hub section and $\Delta r=1$ leads to the tip section. 


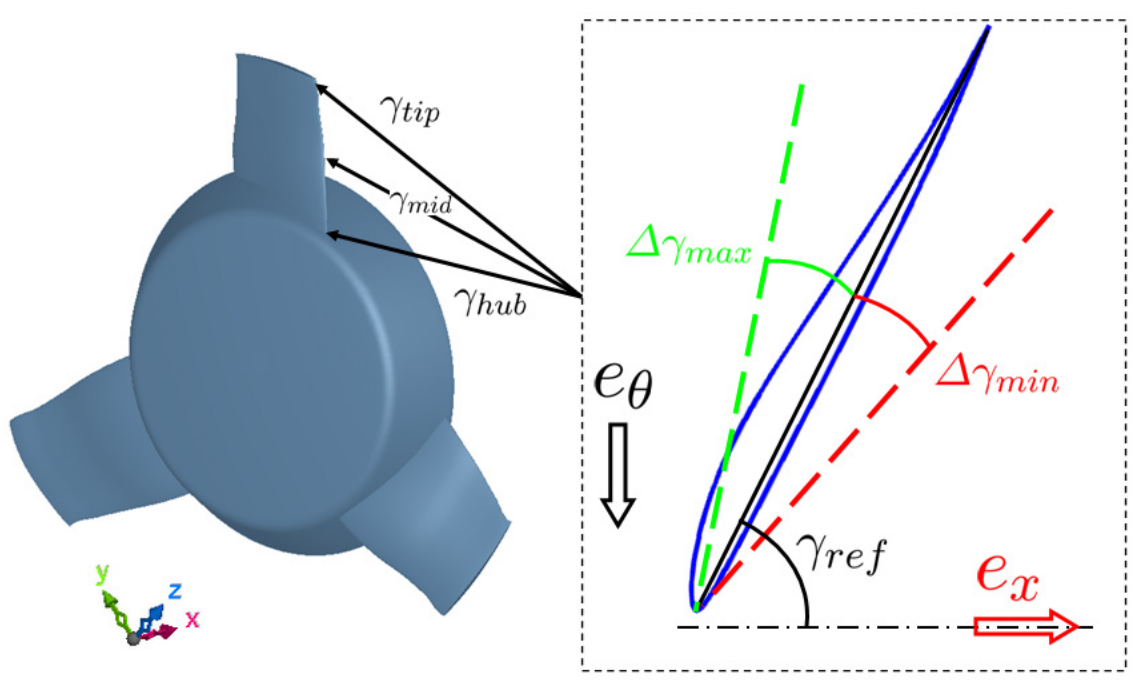

Fig. 1. Parameterized stagger angles $\gamma_{\text {hub }}, \gamma_{\text {mid }}$ and $\gamma_{\text {tip }}$ (left) and the illustration of their variation (right).

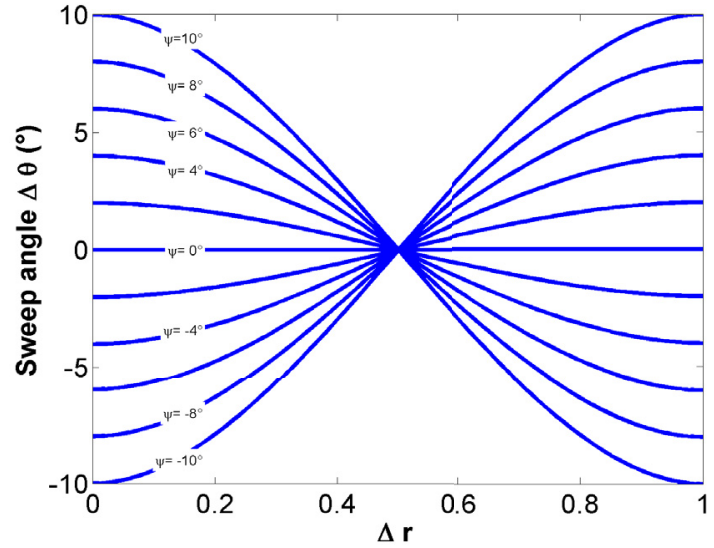

Fig. 2. Sweep function.

The deformation is done as illustrated in Figure 2, where the sweep angle $\Delta \theta\left(^{\circ}\right)$ is presented as a function of the span variation $\Delta r$, with amplitude given by parameter $\psi\left({ }^{\circ}\right)$ and showing point reflection symmetry of center $\left(r=0.5 ; \Delta \theta=0^{\circ}\right)$. Positive values of parameter $\psi\left(^{\circ}\right)$ make the sections close to the hub move forward (same direction as fan rotation direction) and the sections close to the tip move backward (opposite direction of fan rotation direction). Negative values of parameter $\psi\left(^{\circ}\right)$ lead to the inverse deformation. $\psi=0^{\circ}$ means the sweep angle is not modified.

Fan parameterization is based on these four parameters. Surface mesh modifications of the fan are then propagated in the volume mesh through radial basis functions (RBF) and additional constraints are imposed to maintain the shapes of both hub and shroud.

Figure 3 shows all the control sections for the mesh deformation. This latter is done in four steps:

- user input: $n$ control sections are chosen by constantradius cut;

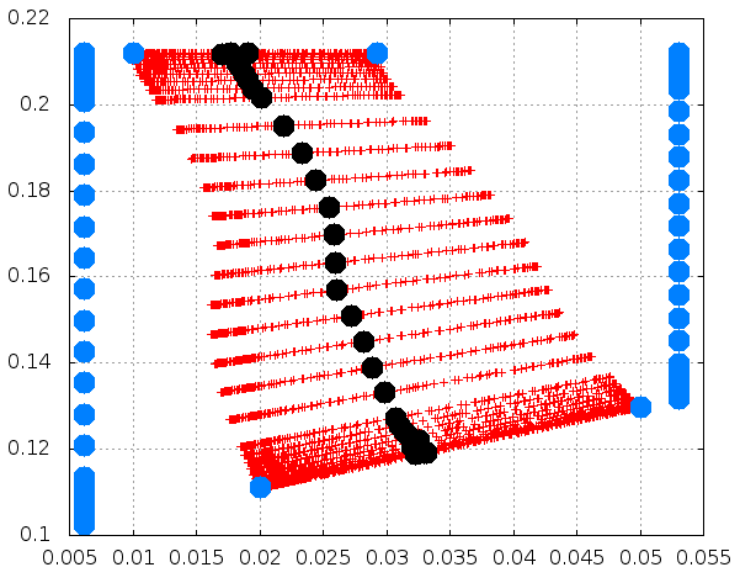

Fig. 3. 2D view of control sections used for mesh deformation.

- user input: three of the selected control sections (hub, mid-span and tip) are driven by the aforementioned parametric parameters. Their displacements are inputted to start the deformation procedure;

- deformation algorithm: displacements of the $n-3$ other sections are calculated by quadratic or cubic interpolation;

- deformation algorithm: the morphing technique is employed to calculate new positions of each node in the volume mesh, using RBF rules and imposed constraints.

An example of a mesh deformation is given in Figure 4.

Figure 4a compares the original surface mesh (left) to the morphed one (right) given $\psi=-8^{\circ}$. A close view of the trailing edge near the hub is presented in Figure $4 \mathrm{~b}$, where it can be observed that the mesh smoothness, refinement level and regularity have been preserved. Compared to traditional CAD parameterization which uses different meshes for different configurations, this method limits CFD uncertainties by morphing from one single 


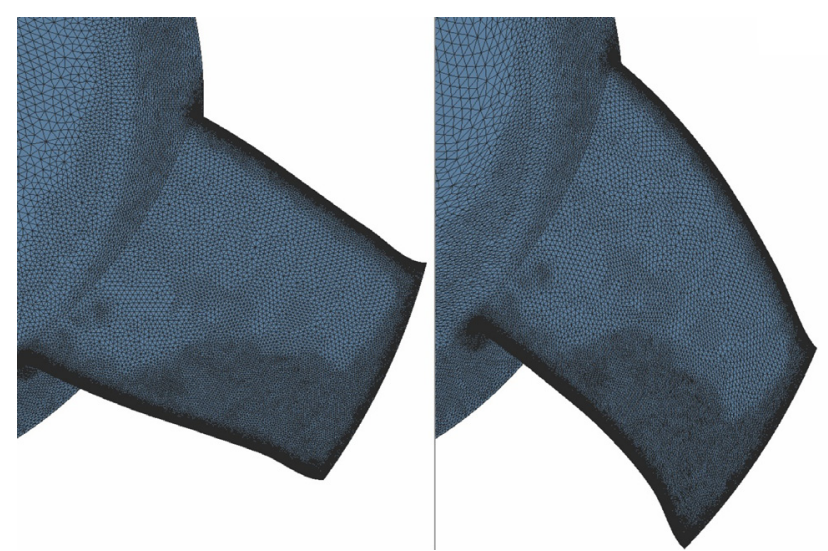

(a) blade surface

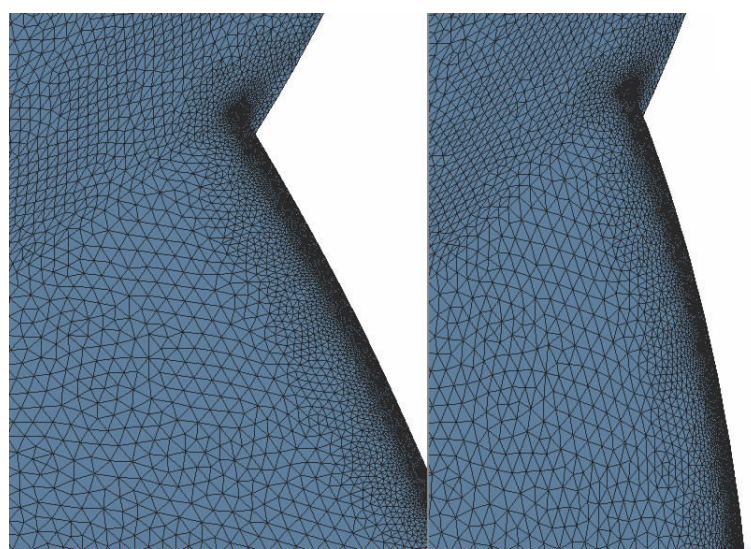

(b) zoom to surface mesh at trailing edge

Fig. 4. Example of deformation: Sweep parameter $\psi=0^{\circ}$ (left) $\psi=-8^{\circ}$ (right).

Table 1. Ranges of extrapolation.

\begin{tabular}{cccc}
\hline$\Delta \gamma_{\mathrm{hub}}\left({ }^{\circ}\right)$ & $\Delta \gamma_{\mathrm{mid}}\left({ }^{\circ}\right)$ & $\Delta \gamma_{\mathrm{tip}}\left(^{\circ}\right)$ & $\psi\left(^{\circ}\right)$ \\
\hline$[-2 ; 2]$ & {$[-4 ; 4]$} & {$[-5 ; 5]$} & {$[-8 ; 8]$} \\
\hline
\end{tabular}

mesh for all the numerical simulations. All the morphed meshes have the same number of elements as the mesh associated with the reference configuration.

The valid range of variation for each parameter has been determined by testing possible mesh deformations and by checking their respective quality. The ranges of all the parameters are given in the Table 1.

Deformation at hub is limited in the range $\left[-2^{\circ} ; 2^{\circ}\right]$ because of constraints on the hub, whereas they are larger at mid-span and tip where there are less interactions with the surrounding. The sweep parameter $\psi$ can be validated up to $\pm 8^{\circ}$, as it is shown in the Figure 4 .

\section{Derivative database and meta-model building}

In this study, an aerodynamic derivative database containing first-order, second-order and second-order cross derivatives of objectives with respect to the geometric parameters is computed using an ordinary least square method. The latter is fuelled with the objectives' values coming from a set of numerical flow simulations.

Figure 5 shows the values of parameters for all the geometric configurations that are numerically simulated when using only two parameters. Mesh morphing for each configuration is required, except for the reference one (where all the parameters' variations are zero).

For each parameter, four CFD samples (black points in Fig. 5) around the reference one (red point in Fig. 5) enable to compute first-order and second-order monoparametric derivatives. Although three CFD samples would be enough to calculate mono-parametric derivatives up to the second order, five CFD samples (including

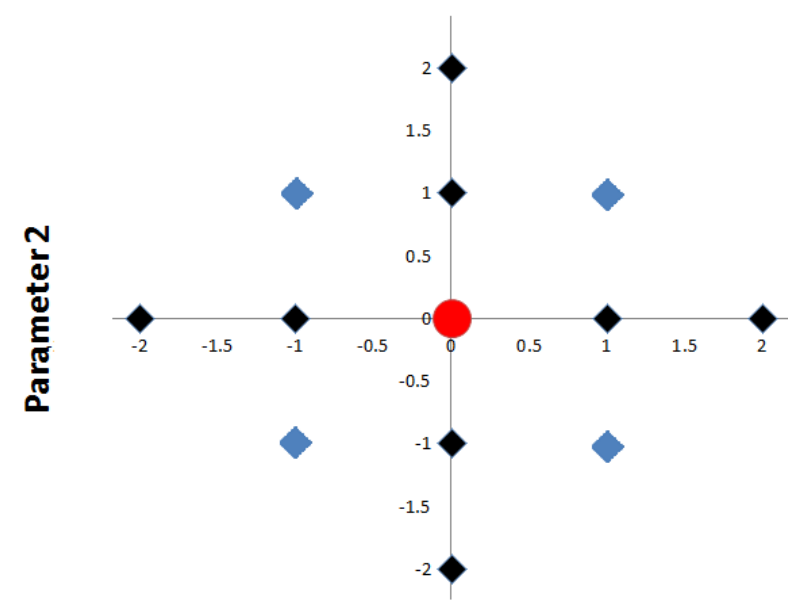

Parameter 1

Fig. 5. CFD sampling used to compute derivatives.

the one associated with the reference configuration) are used here in order to limit the error brought by CFD uncertainties.

Four additional CFD samples, depicted by blue points in Figure 5, accompanied by the previously computed mono-parametric derivatives enable to compute the second-order cross derivative of the objectives with respect to parameters 1 and 2 .

When using more parameters, the same sampling strategy is applied and the final number of samples is given by the formula $2 n \times(n+1)$ where $n$ is the number of parameters. In this study, where four geometric parameters were used, 41 numerical flow simulations were carried out including the one corresponding to the reference configuration.

Parameters' ranges for the derivative database computation are $\left[-2^{\circ} ; 2^{\circ}\right]$ for all the four parameters (Fig. 5). 


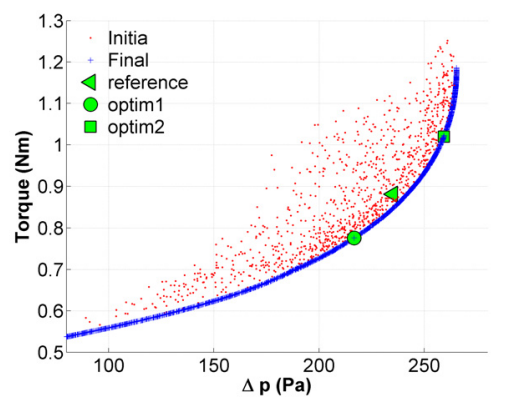

(a) $\Delta P_{s}-\mathrm{T}$

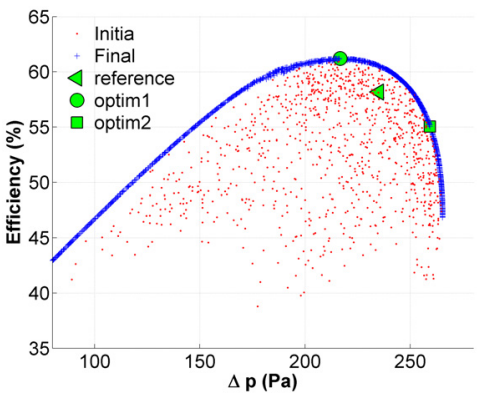

(b) $\Delta P_{s}-\eta_{s}$

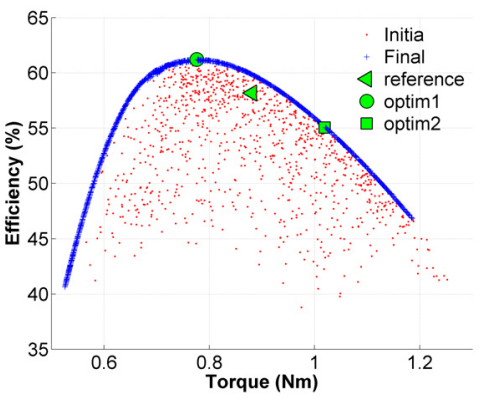

(c) $\mathrm{T}-\eta_{s}$

Fig. 6. 2D views of the Pareto front.

Once the whole derivative database is computed, a meta-model can be built for each objective using a multi-parameter high-order Taylor-series expansion:

$F(p+\Delta p)=F(p)+F^{(1)} \Delta p+\ldots+\frac{F^{(n)}}{n !} \Delta p^{n}+o\left(\Delta p^{n+1}\right)$

where $F(p+\Delta p)$ is the objective reconstructed in term of a variation $\Delta p$ of the parameter $p$. In Equation (3), the truncation error is of the magnitude of $\Delta p^{n+1}$. A metamodel is built for each of the three following objectives:

- the static pressure rise $\Delta P_{s}$, defined by the static pressure difference between inlet and outlet of the computational domain:

$$
\Delta P_{s}=P_{s}^{\text {out }}-P_{s}^{\text {in }}
$$

where $P_{s}^{\text {out }}$ and $P_{s}{ }^{\text {in }}$ are the static pressure at outlet and inlet respectively;

- the torque $T$, defined by the axial moment of pressure force $\vec{F}_{p}$ and viscous force $\vec{F}_{v}$ acting on the blades:

$$
T=\int\left(\overrightarrow{F_{p}}+\overrightarrow{F_{v}}\right) \times \overrightarrow{e_{r}} \mathrm{~d} r
$$

where $\overrightarrow{e_{r}}$ is the radial unit vector, $r$ is the radius scalar;

- the static efficiency $\eta_{s}$, defined by the ratio of the aerodynamic power and the mechanical power provided by electrical motor:

$$
\eta_{s}=\frac{\Delta P_{s} \times Q}{T \times N}
$$

where $Q$ is the volume flow rate, and $N$ is the fan rotation speed.

\section{Multi-parameter multi-objective optimization results}

Meta-models are explored with a genetic algorithm optimizer. The second version of Non-dominated Sorting Genetic Algorithm (NSGA-2) proposed by Deb et al. [3] has been applied for this purpose. Projected views of the Pareto front are presented in Figure 6.
The red dots are individuals of the first generation, and the blue line represents all Pareto optimal solutions, given by the NSGA-2 algorithm. As an indication, the reference point is represented by the triangle, and its performances are $235.1 \mathrm{~Pa}$ for pressure rise, $0.8808 \mathrm{Nm}$ for torque and $58.16 \%$ for efficiency. It should be noted that this design is very close to the Pareto front. Two different optimal points have been retained, which could be solutions for different customer specifications. The first one, named optim 1, is depicted as a filled circle in Figure 6 and has been chosen because it exhibits the highest value of static efficiency on the Pareto front while maintaining sufficient static pressure difference level. The $\Delta P_{s}$ at this point is about $18 \mathrm{~Pa}$ lower than the reference, but its efficiency is increased by three points, from $58.16 \%$ to $61.21 \%$, and a significant reduction of torque is also obtained. The second optimal solution, optim 2, is shown as a filled square in Figure 6 and has been selected because it maximizes the static pressure difference while keeping a static efficiency greater than $55 \%$.

Self-Organizing Maps (SOM) [4] can be used to make the connection between design space and objectives' space effectively. Self-organizing maps are a type of neural network designed to understand high-dimensional data with help of its low-dimensional representations [5]. Each variable (parameter or objective) is depicted as a twodimensional map which preserves topological properties of the Pareto optimal solutions. One individual is always found at the same $2 \mathrm{D}$ position from one map to another.

In the Figure 7, there are seven maps which represent the values of four parameters (four maps on top) and three objectives (three maps below) on the Pareto fronts.

It is shown on those SOM the two optima by a round dot (optim 1) and a square dot (optim 2). With the help of those maps, we can have a global view of the parameter values taken by optim 1 , so that the variations of hub stagger angle, mid-span stagger angle and tip stagger angle are respectively $2^{\circ}, 2.4^{\circ}$ and $-2^{\circ}$, and sweep angle is zero. Compared with the reference blade, the optim 1 configuration is more staggered at the hub and at mid-span, less-staggered at the tip and its sweep angle is identical. Its objective values are $216.7 \mathrm{~Pa}$ for $\Delta P_{s}, 0.7762 \mathrm{Nm}$ for torque, and $61.21 \%$ for efficiency. Hence, optim 1 is a suitable low-torque and high-efficiency design. 

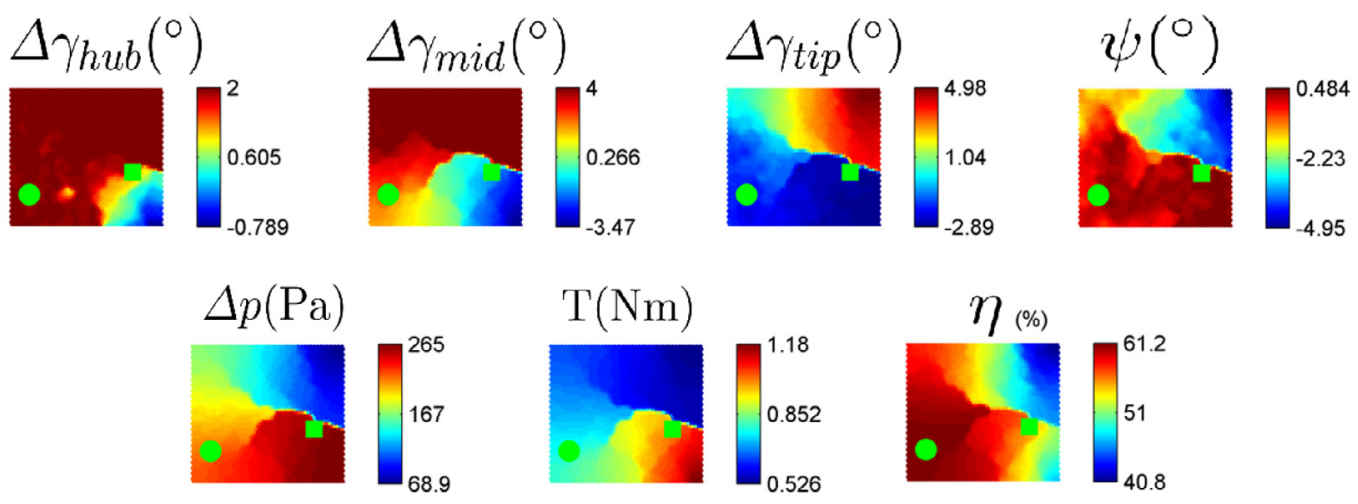

Fig. 7. Visualization of the Pareto front with help of SOM.

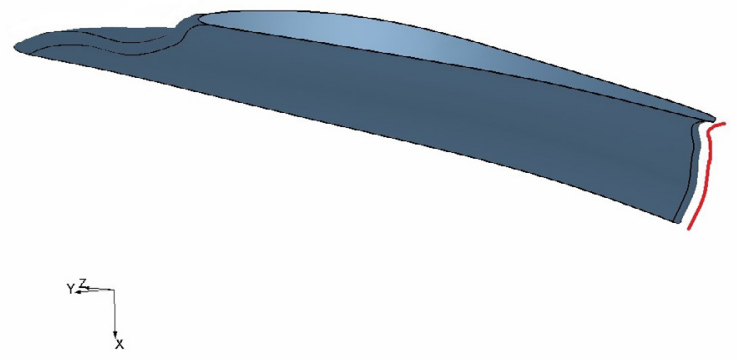

Fig. 8. Velocity measurement position (4 $\mathrm{mm}$ downstream away from the red curve).

The values taken by optim 2 are $1.18^{\circ},-1.43^{\circ},-2.98^{\circ}$ and $0.43^{\circ}$ for the variations of three stagger angles and $\psi$ respectively, with the constraint on efficiency of $55 \%$, the optim 2 has a $\Delta P_{s}$ of $259.3 \mathrm{~Pa}$ and a torque of $1.02 \mathrm{Nm}$ according to meta-model. As a consequence, optim 2 is more appropriate for higher pressure demands.

In the following, the authors focus on the aerodynamic analysis of optim 1 and partly explain how the improvements on global performance brought by this new configuration are related to local changes in the aerodynamic flow field.

In the Figure 8, a curve located at downstream the blade trailing edge is used to measure the circumferential average of axial, radial and tangential components of absolute velocity, from hub to tip. Comparisons, based on these three latter variables, between the reference configuration and the optim 1 configuration are achieved in Figures 9-11, respectively.

As far as the axial velocity is concerned (Fig. 9), the reference and the optim 1 configurations have parallel evolutions at low radius, the only difference being the level of this variable which is slightly lower for optim 1 . The peak axial velocity of optim 1 is consequently less important

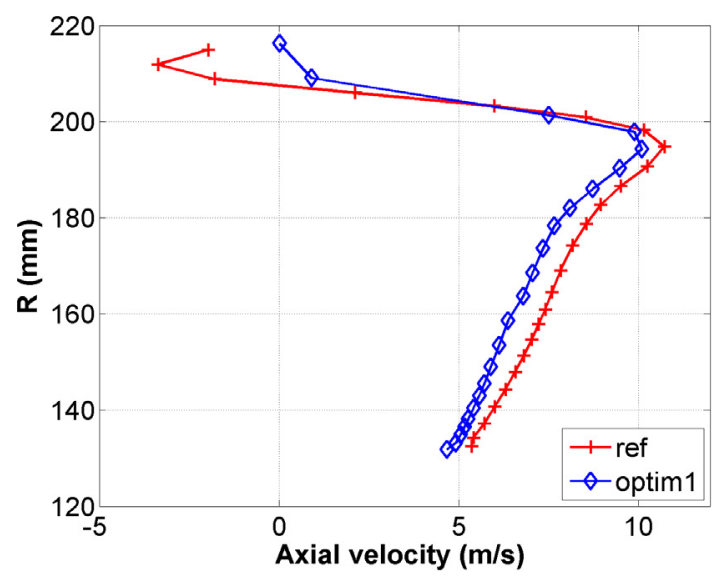

Fig. 9. Circumferentially averaged axial velocity.

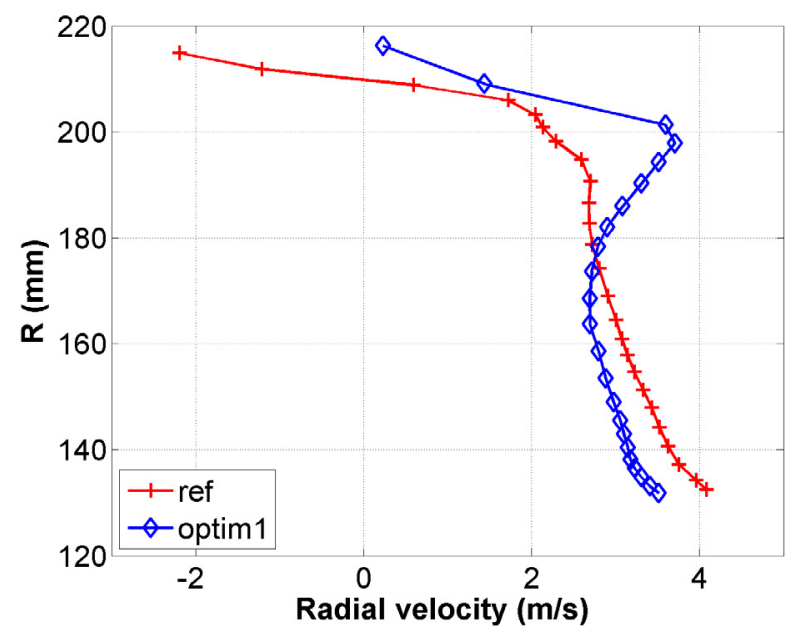

Fig. 10. Circumferentially averaged radial velocity.

than the one of the reference. Significant differences rather appear in the tip gap, where the negative value for the reference curve indicates a tip-leakage flow. This latter has disappeared after the optimization. 
Table 2. Validation of optimizations by CFD simulations.

\begin{tabular}{cccccccc}
\hline & \multicolumn{3}{c}{ Design parameters } & \multicolumn{3}{c}{ Objectives } \\
\cline { 2 - 7 } & $\Delta \gamma_{\mathrm{hub}}\left({ }^{\circ}\right)$ & $\Delta \gamma_{\mathrm{mid}}\left({ }^{\circ}\right)$ & $\Delta \gamma_{\mathrm{tip}}\left(^{\circ}\right)$ & $\psi\left(^{\circ}\right)$ & $\Delta P_{s}(\mathrm{~Pa})$ & $T(\mathrm{Nm})$ & $\eta_{s}$ \\
\hline Reference & 0 & 0 & 0 & 0 & 235.1 & 0.8808 & $58.16 \%$ \\
Range explored & {$[-2 ; 2]$} & {$[-4 ; 4]$} & {$[-5 ; 5]$} & {$[-8 ; 8]$} & - & - & - \\
Optim 1 & 2.0 & 2.4 & -2.0 & 0 & 216.7 & 0.7762 & $61.21 \%$ \\
CFD validation of optim 1 & 2.0 & 2.4 & -2.0 & 0 & 216.3 & 0.7668 & $61.47 \%$ \\
Relative error of optim 1 & - & - & - & - & $0.18 \%$ & $1.20 \%$ & $-0.42 \%$ \\
Optim 2 & 1.18 & -1.43 & -2.98 & 0.43 & 259.3 & 1.0194 & $55.03 \%$ \\
CFD validation of optim 2 & 1.18 & -1.43 & -2.98 & 0.43 & 254.3 & 0.9955 & $55.67 \%$ \\
Relative error of optim 2 & - & - & - & - & $1.97 \%$ & $2.40 \%$ & $-1.15 \%$ \\
\hline
\end{tabular}

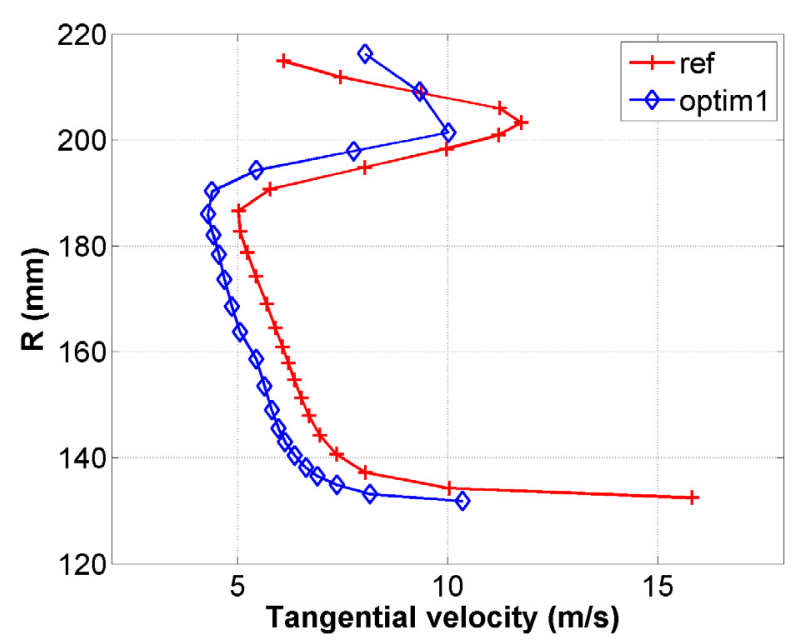

Fig. 11. Circumferentially averaged tangential velocity.

Considering now the radial velocity (Fig. 10), it can be seen that the curves of the two geometries intersect at about $R=175 \mathrm{~mm}$. For higher radius values, radial velocity observed on the reference configuration keeps on decreasing till approximately $-2 \mathrm{~m} . \mathrm{s}^{-1}$, whereas radial velocity increases till roughly $4 \mathrm{~m} . \mathrm{s}^{-1}$ for optim 1 before dropping to zero. Radial velocity stays positive for the optim 1 geometry which indicates that the downstream recirculation is absent.

Figure 11 shows the comparison of tangential velocity profiles. The reference configuration is characterized by higher tangential velocity values almost all along the radius compared with optim 1 , meaning that the reference fan exerts more work on the air flow. This is consistent with the pressure rise produced by the reference configuration.

The results of optimization are summarized in Table 2, where results from the meta-model and CFD simulations are presented. Differences between them are shown by their relative errors.

The computation has confirmed performance for optim 1 with a slightly lower pressure rise $(0.4 \mathrm{~Pa})$, lower torque $(0.0094 \mathrm{Nm})$ and higher efficiency (0.26 points). The same tendency is found for optim 2 .
Comparison between meta-models results and CFD results for the two optima shows small relative errors, with a maximum observed error of about $2 \%$ for pressure rise, $2.4 \%$ for torque and $1.15 \%$ for efficiency. The errors on the two optima can have mainly three causes:

- Uncertainties of CFD simulation because of mesh quality variation (aspect ratio and skewness vary with the deformation).

- The derivative computation errors brought by the ordinary least squares method.

- Truncation error, since derivatives up to second order are used to construct the meta-model, second order truncation error is present by nature.

The overall error level is under $2.5 \%$, which suggests that the optimization process is reliable, and future experimental tests could validate this approach.

\section{Conclusion}

A new concept engine cooling fan characterized by a conical hub was studied with a meta-model based multiobjective optimization tool assisted by a mesh morphing technique. Three global performance objectives and four geometric parameters were taken into consideration during the optimization process. From a reference design, derivatives up to second order were calculated with ordinary least squares method. Polynomial meta-models for three objectives were built based on those derivatives. The meta-models were explored using NSGA-2, and two optima had been extracted from the Pareto front. One of the optima was found to be able to raise the efficiency by three points, and to reduce significantly the torque which is the main factor that determines the cost of the electrical motor. The other optimum met higher pressure rise needs as it exhibited an increase of $29 \mathrm{~Pa}$, with a moderate loss of 2.5 points on efficiency. Therefore, this configuration is better suited to high heat exchange between air and the radiator.

Acknowledgements. The current study is part of a project of VALEO powertrain thermal systems group, it is strongly supported by the research team of FLUOREM S.A.S. The authors gratefully acknowledge Cradle Co., LTD for implementing the software SC/Tetra. 


\section{References}

[1] L. Soulat, P. Ferrand, S. Aubert, S. Moreau, M. Henner, Numerical design and optimization of a new casing treatment for shrouded fans, 27th Congress of International Council of the Aeronautical Sciences, September 2010, Nice, France

[2] M. Buisson, P. Ferrand, L. Soulat, S. Aubert, C. Rambeau, M. Henner, Optimal Design of an Automative Fan using the Turb'Opty Metamodel, J. Comput. Fluids in Press, online 30 March, 2012

[3] D. Kalyanmoy, S. Agrawal, A. Pratap, T. Meyarivan, A fast elitist non-dominated sorting genetic algorithm for multi-objective optimization: NSGA-II, In Parallel Problem Solving from Nature PPSN VI, Vol. 1917, 2000, pp. 849-858. Springer, Berlin, Heidelberg

[4] J. Vesanto, J. Himberg, E. Alhomieni, J. Parhankangas, SOM toolbox for matlab5, Report A57, Helsinki University of Technology, 2000

[5] L. Soulat, P. Ferrand, S. Moreau, S. Aubert, M. Buisson, Efficient optimization procedure for design problems in fluid mechanics, J. Comput. Fluids 82 (2013) 73-86

[6] M. Buisson, C. Rambeau, P. Ferrand, S. Aubert, L. Soulat, M. Henner, Parameters Coupling Effects on 2D Optimal
Design of a Fan Blade, 10th International Symposium on Experimental and Computational Aerothermodynamics of Internal Flows, July 2011, Bruxelles, Belgium

[7] M. Buisson, P. Ferrand, L. Soulat, S. Aubert, S. Moreau, C. Rambeau, M. Henner, Turb'Opty Based Optimization Of A Shrouded Fan With Casing Treatment 28th International Congress Of The Aeronautical Sciences, pp. 23-28, 2012, Brisbane, Australia

[8] M. Henner, S. Moreau, J.-F. Brouckaert, Comparison of experimental and numerical flow field in an automotive engine cooling module, 8th European Conference on Turbomachinery Fluid Dynamics and Thermodynamics, 2009, pp. 23-27, Graz, Austria

[9] D. Monier, F. Gillot, M. Ichchou, Integrated workflow for multi-objective evolutionary optimization of the vehicle tyre parameters, J. Automobile Eng. 227 (2013) 222-233

[10] Y. Rozenberg, S. Aubert, G. Benefice, Fluid structure interaction problems in turbomachinery using RBF interpolation and greedy algorithm, Proceedings of ASME Turbo Expo 2014, pp. 16-20, Dusseldorf, Germany

[11] A. Saltelli, M. Ratto, T. Andre, Global sensitivity analysis, Wiley, 2008 